\title{
Semiconductor chip's quality analysis based on its high dimensional test data
}

\author{
Sun $\mathrm{Kai}^{1} \cdot \mathrm{Wu} \mathrm{Jin}^{1}$ \\ Published online: 14 May 2019 \\ (c) The Author(s) 2019
}

\begin{abstract}
A semiconductor chip usually has thousands test parameters in order to guaranteed its quality. Hence, a batch of chips' test data set include thousands of float data. The primary goal of dealing with this test data is to obtain the fault parameter distribution and judge the chip's quality. It is a challenge due to the large scale and complex relationship of the test data set. This paper presents a novel method to analyze the test data set by meshing the quality theory and scientific data visualization. First, transfer the test data set to a quality classifier matrix Q: a series of quality region is defined based on quality theory, which is the baseline to classify the test data set into different group and mark them with various number. Second, form a qualityspectrum: define a color rule based on the RGB color model and color the quality classifier matrix Q. Hence chip's quality distribution could be observed through the quality-spectrum. Furthermore, by analyzing the quality-spectrum, the chip's quality could be quantitative and fault diagnose has a data basic. One case is included to illustrate appropriateness of the proposed method.
\end{abstract}

Keywords Data processing · Quality-spectrum · Industrial electronics · Quality control

\section{Introduction}

Chip's quality is crucial to a semiconductor industry. In order to guarantee the chip's quality, thousands of test parameters have been defined. Besides, it always includes hundreds even thousands of chips in one test process. Hence, a batch of chip's test data includes at least hundreds even thousands of float data. The units of these test parameters are various. And the relationship among these test parameters are complex. So the test data set represents a

$\bowtie$ Wu Jin

wujin@ime.ac.cn

Sun Kai

sunkai1@ime.ac.cn

1 Microwave Device and Integrated Circuits Laboratory, Institute of Microelectronics of Chinese Academy of Sciences, Beijing 611731, People's Republic of China 
typical multivariable nonlinear coupling system. The useful information, such as the chip's quality, the relationship among the fault parameters, the fault distribution etc. is hidden inside the "data sea". It's difficult to deal with the test data set and obtain it. Essentially, obtaining useful information from test data set is a problem of nonlinear multivariate series analysis, which is a challenge for decades.

The conventional data-driven approaches of the process monitoring are multivariate statistics analysis (MSA) (Amin et al. 2018; He and Tan 2018; Tootooni et al. 2018; Jiang et al. 2017), support vector machines (SVM) (Nguyen and Lee 2018; Du et al. 2018; Ma et al. 2017; Galmeanu et al. 2016)and inductive data mining (IDM). The partial least squares (PLS) (Nomikos and MacGregor 1995; Dong et al. 2015; Foo et al. 2018) and principal component analysis (PCA) (Wold et al. 1987; Gharahbagheri et al. 2017; Dong and Qin 2018; Bakdi and Kouadri 2018; Burgas et al. 2018; Cai and Zhang 2016) are the representative techniques of MSA. Both methods are challenged by the process of non-Gaussian distribution and nonlinear multivariate time series. Independent component analysis (ICA) is focus on dealing with non-Gaussian characteristics (Lee et al. 2004; Lee et al. 2006; Wang et al. 2012; Habchi et al. 2018). Neural-network-related PCA (NN-PCA) (Chen and Liao 2002; Dong and McAvoy 1996), kernel principal component analysis (KPCA) (Alcala and Qin 2010; Ge et al. 2009; Lee et al. 2004; Lee et al. 2007; Mania Navi et al. 2018; Wang et al. 2017; Arroyo-Hernandez 2016) and kernel independent component analysis (KICA) (Zhang 2008; Dyer et al. 2017; Li et al. 2016) could be applied to solve nonlinear problem. SVM (Jemwa and Aldrich 2005; Zeng et al. 2006) establishes a robust non-linear model to offer accurate quantitative predictions in classification.

These common methods reduce the dimension of its input variables. The first step of these methods is to select 'primary' and 'useful' elements and omit others. Is it true that the omitted elements is useless? The investigation shows that most chip's fault is rising from insignificant equipment. It is difficult to select a appropriate function to choose "kernel" parameters. Therefore, all the data should be analyzed simultaneously in order to get accurate operating state of the system.

Therefore, it is a challenge to find an appropriate method dealing with huge, nonlinear and multivariate time series simultaneously and efficiently. All the field that require dealing with high dimensional nonlinear series data set face the same challenge. In the field of process industry, a color-spectrum is defined by coloring the monitor data set with RGB color mode(Kai et al. 2015; Gao et al. 2016). This method has the advantage of displaying overall data set on one picture. That is, it avoids select "primary" parameter. Regardless the amount of the parameters, each parameters has the same significance and could be taken into account through analyzing the color-spectrum.

Hence,considering the special requirement of the semiconductor chip's quality analysis, the method of color-spectrum is developed. By combining the theory of Shewhart-type control chart (Tong et al. 2004; Djauhari et al. 2017; Iglesias et al. 2016) and scientific data visualization (Montgomery 2005; Chu and Wu 2016), a new method named quality-spectrum is proposed. As we know, the chip's data set is a huge, nonlinear and multivariate series that contains all information of the chip's quality. The primary aim for analyzing the test data set is to explore the distribution of its abnormal data. The advantage of Shewhart-type control chart is classify data into different regions. Meanwhile, scientific visualization could display the unseen information hidden inside the data sea on a picture (Brodlie et al. 1992). Through analyzing the statistical characteristics of each series individually, Shewhart-type control chart limits the value region named in Q_region for the whole test data set. Hence, it separates the test data set into several groups from worst to best. The quality-spectrum is formed by coloring different groups with various color. By this way, a complicate float data 
set is transferred to a simple digital color image. The distribution of the chip's quality could be obtained qualitatively by observing the quality-spectrum.

The outline of this paper is the following: In Sect. 2, a matrix Q_region representing different quality degree filters is defined. In Sect. 3: The definition of quality-spectrum is proposed. In Sect. 4, one case has been used to verify the validation of the methods. This paper is closed with a conclusion in Sect. 5.

\section{Quality classified matrix}

Suppose a chip's quality including $n$ test parameters. A test batch includes $m$ chips. Hence test data set of the batch is a $m \times n$ float data matrix.

Definition 1. A batch of chip's test data could be arranged as a test Data Matrix X. It's a $m \times n$ matrix as Eq. 1 shows.

$$
X=\left[\begin{array}{cccccc}
x_{11} & x_{12} & \cdots & \cdots & \cdots & x_{1 n} \\
x_{21} & x_{22} & \cdots & \cdots & \cdots & x_{2 n} \\
\vdots & \vdots & \ddots & \cdots & \cdots & \vdots \\
\vdots & \cdots & \cdots & x_{i j} & \cdots & \vdots \\
\vdots & \vdots & \vdots & \vdots & \ddots & \vdots \\
x_{m 1} & x_{m 2} & \cdots & \cdots & \cdots & x_{m n}
\end{array}\right]_{m \times n} \quad \text { Chip' s I D }
$$

In Eq. 1 each element $x_{i j}\left(x_{i j} \in X, 1 \leq i \leq m, 1 \leq j \leq n, i, i \in N\right)$ is a particular test data, where $i$ represents the chip's ID and $j$ marks testing parameter.

Considering each test parameter has been defined a perfect value. A chip best quality should be defined by a $1 \times n$ vector named Perfect_vector as Eq. (2) shows.

$$
\begin{gathered}
\text { Parameters } \\
\text { Perfect_vector }=\left[\begin{array}{llllll}
\theta_{1} & \theta_{2} & \cdots & \theta_{j} & \cdots & \theta_{n}
\end{array}\right]_{1 \times n}
\end{gathered}
$$

Obviously, perfect_vector is an ideal quality value for the test data which is the baseline of real test data. Therefore, it formed a tolerate region based on the perfect_vector. One is an Up_quality_vector as Eq. (3) shows.

$$
\begin{gathered}
\text { Parameters } \\
\text { Up_quality_vector }=\left[\begin{array}{llllll}
\theta_{1}+\gamma_{1} & \theta_{2}+\gamma_{2} & \cdots & \theta_{j}+\gamma_{j} & \cdots & \theta_{n}+\gamma_{n}
\end{array}\right]_{1 \times n}
\end{gathered}
$$

The other is a Low_quality_vector as Eq. (4) shows.

$$
\begin{aligned}
& \text { Parameters } \\
& \text { Low_quality_vector }=\left[\begin{array}{llllll}
\theta_{1}-\delta_{1} & \theta_{2}-\delta_{2} & \cdots & \theta_{j}-\delta_{j} & \cdots & \theta_{n}-\delta_{n}
\end{array}\right]_{\times n}
\end{aligned}
$$

Based on Eq. (3) and Eq. (4), the perfect quality chips's baseline follows the Eq. (5).

$$
X_{\text {Perfect }}: \theta_{j}-\delta_{j}<x_{i}^{j}<\theta_{j}+\gamma_{j}, x_{i}^{j} \in X
$$




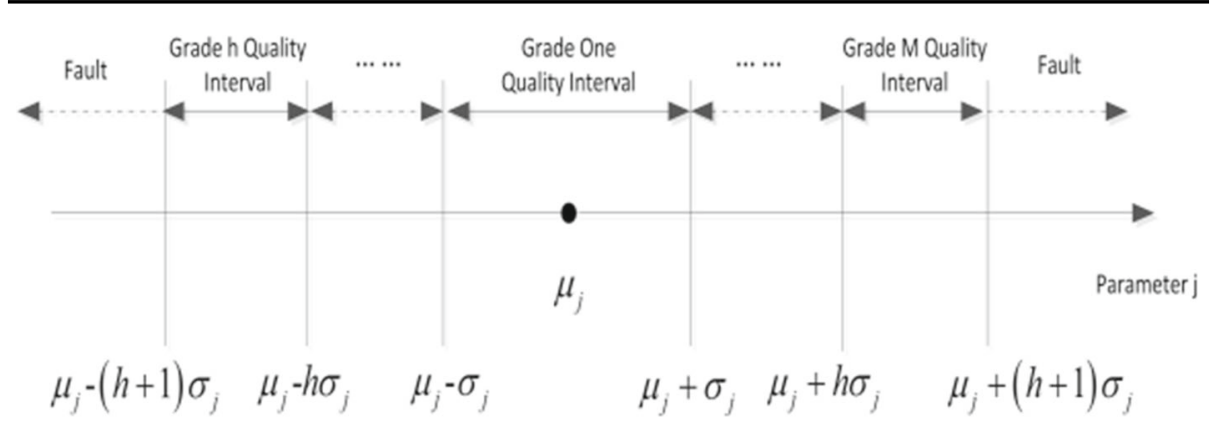

Fig. 1 The quality region of parameter $\mathrm{j}$

Suppose $k$ chips are suitable to the baseline, a $k \times n$ perfect data set $X_{\text {Perfect }}$ is formed as Eq. 6 shows.

$$
X_{\text {Perfect }}=\left[\begin{array}{cccccc}
x_{11} & x_{12} & \cdots & \cdots & \cdots & x_{1 n} \\
x_{21} & x_{22} & \cdots & \cdots & \cdots & x_{2 n} \\
\vdots & \vdots & \ddots & \cdots & \cdots & \vdots \\
\vdots & \cdots & \cdots & x_{i j} & \cdots & \vdots \\
\vdots & \vdots & \vdots & \vdots & \ddots & \vdots \\
x_{k 1} & x_{k 2} & \cdots & \cdots & \cdots & x_{k n}
\end{array}\right]_{k \times n} \text { Chip' s I D }
$$

Each column $x_{j} \in X_{\text {Perfect }}$ is a vector of $k$ chip's test data of parameters $j$. The average value of each column means the benchmark parameter $j$. And the standard deviation of each column is used to calculate the distant of the test data away from its benchmark.

Definition 2. Chip_E is $1 \times n$ vector composed with $n$ parameters' benchmark as Eq. 7 shows.

\section{Parameters}

$$
\text { Chip }_{-} E=\left[\begin{array}{llllll}
\mu_{1} & \mu_{2} & \cdots & \mu_{j} & \cdots & \mu_{n}
\end{array}\right]_{1 \times n}
$$

Where $\mu_{j}\left(\mu_{j}=\frac{1}{n} \sum_{i=1}^{k} x_{i j}, x_{i j} \in X_{\text {Perfect }}\right)$ is the mean of parameter $j$.

Definition 3. Chip_Std is one dimensional vector of each parameters' standard deviation based on the benchmark data matrix $X_{\text {Perfect }}$ as Eq. 8 shows

\section{Parameters}

$$
\text { Chip_Std }=\left[\begin{array}{llllll}
\sigma_{1} & \sigma_{2} & \ldots & \sigma_{j} & \ldots & \sigma_{n}
\end{array}\right]_{1 \times n}
$$

where $\sigma_{j}=\sqrt{\frac{1}{n-1} \sum_{i=1}^{k}\left(x_{i j}-\mu_{j}\right)^{2}}$ is the standard deviation of sensor $j$.

The chip's quality could be separated into $h$ degree. For each chip's test parameter, it has $h$ quality grade interval between the perfect and fault. Figure 1 shows the arrangement of the quality grade interval for test parameter $\mathrm{j}$.

As Fig. 1 shows, the parameter j's deviation from its mean is the acknowledged quality standard based on the theory of Shewhart control chart. By this way, the chip's quality region based on the test data set could be defined. 


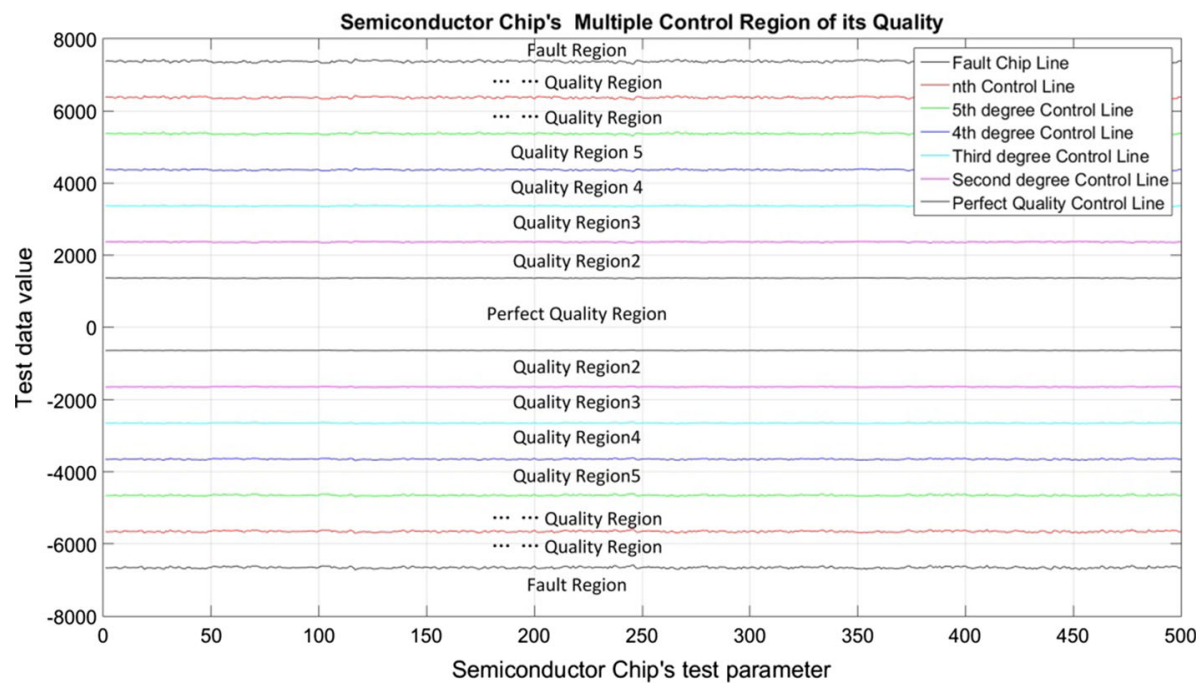

Fig. 2 Semiconductor chip's multiple quality region

Definition 4. Q_Region is a matrix enclosed overall test parameters' upper and lower control limits of as Eq. 9 shows.



In Eq. (9), $h$ is used to indicate the number of the quality region. Q_Region gives the quality lines that could classify the chip's test data set, as Fig. 2 shows.

By calculating the mean and standard deviation of each parameter based on the benchmark matrix $X_{\text {Perfect }}$, the quality classify standard Q_Region could be obtained. As we discussed above, a batch of semiconductor chip's test data set is a data matrix $X$ which including $m \times n$ test data. The elements of data matrix $X$ is float. Generally, $n$ which represents the number of test variables is always at the quantity of thousands. The elements of the test data set could be classified using Q_Region as a filter. The quality of the chip is divided into $h$ categories following the definition of the Q_Region as Eq. (5) shows. Hence the float test data set has been transferred to an integer quality classifier matrix Q, as Eq. (6) shows.

$$
\begin{aligned}
& \text { Test Parameter }
\end{aligned}
$$




Fig. 3 RGB color cube

Table 1 Color table

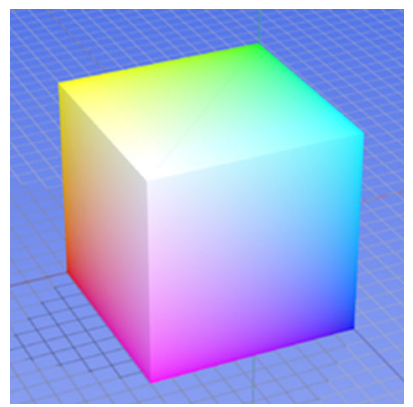

\begin{tabular}{lrrrl}
\hline Q_region & R & G & B & Color \\
\hline 0 & 0 & 255 & 0 & Green \\
1 & 255 & 255 & 0 & Yellow \\
2 & 255 & 255 & 255 & White \\
3 & 0 & 0 & 255 & Blue \\
4 & 255 & 0 & 255 & Cyan \\
5 & 0 & 0 & 0 & Black \\
6 & 255 & 0 & 0 & Red \\
\hline
\end{tabular}

As Eq. (10) shows, the data matrix $X$ has been marked with special number. The data matrix has been transferred to quality classifier matrix $\mathrm{Q}$.

\section{Chip's quality-spectru}

\subsection{Form quality-spectrum}

Since human's eyes are more sensitive to the color change than the float number, it is easy to observe the distribution of the chip's quality by coloring the quality classifier matrix $\mathrm{Q}$. Visualizing the data values using color corresponds to mapping a single parameter distribution to color(McCormick et al. 1987). The advantage of color expression is that the number of noticeable differences is high. The $R G B$ color model, which is a typical color model, is composed of three basic color: Red, Green and Blue. It is a three-dimensional color phase space $R \times G \times B \in C^{3}$.Fig. 3 shows the a color cube of RGB whose horizontal x-axis as red values increasing to the left, $y$-axis as blue increasing to the lower right and the vertical $z$-axis as green increasing towards the top. The original point of $(\mathrm{r}, \mathrm{g}, \mathrm{b})$ is black $(0,0,0)$ which is the vertex hidden from view. Since the elements of $\Pi$ is the color index corresponding with the data matrix $X$, it is can be colored according with the the RGB color cube.

Based on the theory of data visualization and RGB color cube, a color table $\Pi \in C^{3}$ is defined to coloring the quality classifer matrix Q, as Table 1 shows.

The color rule is $\chi: x_{i j} \rightarrow \Pi \subset C^{3}$, which mapping a sample value $x_{i j} \in Q$ to the color phase space $\Pi \subset C^{3} \cdot \Gamma$ is generated by rendering the color table $\Pi$ mapped data matrix $p_{i j} \in \Pi, i \in[1, m], j \in[1, n]$ by using special RGB model $\xi$ as Eq. 11 shows.

$$
\xi: Q \times \Pi \rightarrow \Gamma
$$


Definition 5. Quality-spectrum $\Gamma$ is a two binary digital image which represents the abnormal monitor data distribution as Eq. 12 shows.

$$
\Gamma=\left\{\text { Pixel_color }_{i j} \mid \text { Pixel_color }{ }_{i j}=\left\{\begin{array}{c}
\text { Green } x_{i j}=0 \\
\text { Yellow } x_{i j}=1 \\
\text { White } x_{i j}=2 \\
\text { Blue } x_{i j}=3 \\
\text { Cyan } x_{i j}=4 \\
\text { Black } x_{i j}=5 \\
\text { Red } x_{i j}=6
\end{array}, \forall x_{i j} \in Q, i \in[1, m], j \in[1, n]\right\}\right.
$$

The quality-spectrum defined Green as the first level, which represents the perfect quality of the chip. And the red means the worst quality that absolutely unacceptable. Yellow, white, blue, Cyan, black represents the quality level decreased from the extreme perfect to totally fail. The quality state of the chip could observed intuitively by converting the test data matrix to a quality-spectrum.

\subsection{Fault distribution of parameters}

There are hundreds of test parameters for each chip. The impact of each parameter to the chip's quality is the root for fault tracing and quality improving. Since the quality classifier matrix Q represents the fault degree each chip's special parameter, the fault score of each parameter is obtained as Eq. (13) shows.

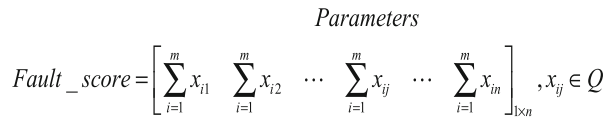

As Eq. (13) shows, the fault score of each parameter is the summary of each column of quality classifier matrix Q.

\subsection{Quantitative chip's quality}

The chip's quality is determined by its test parameters. Suppose each test parameter has the same impact to the chip's quality. The fault score of the chip's quality is obtained by summarizing its overall test parameter's quality score as Eq. (14) shows.

$$
\begin{gathered}
\text { Chip's ID } \\
\text { Fault_score }=\left[\sum_{j=1}^{m} x_{1 j} \sum_{j=1}^{m} x_{2 j} \cdots \sum_{j=1}^{m} x_{i j} \cdots \sum_{j=1}^{m} x_{n j}\right]_{\mathrm{l} \times m}^{T}, x_{i j} \in Q
\end{gathered}
$$

Therefore, the chip's quality could be calculated quantitatively by its fault score. The lower of a chip's fault score represents the better of the chip's quality.

\section{Use case}

A batch of chip's test data looks like Table 2. The number of test chip are thousands as well as its test parameters are over hundreds. Furthermore, the data is float. It is difficult to judge the quality of the chip through the Table 2 directly. 
Table 2 Chip's test data set

\begin{tabular}{|c|c|c|c|c|}
\hline Chip no. & Parameters 1 & Parameter 2 & Parameter 3 & $\ldots$ \\
\hline A001 & -37.3807 & 37.8406 & 5.56227 & $\ldots$ \\
\hline A002 & -38.3323 & 39.5301 & 5.28495 & $\cdots$ \\
\hline A003 & -27.419 & 27.6164 & 4.26187 & $\ldots$ \\
\hline A004 & -39.272 & 41.1711 & 5.73834 & $\ldots$ \\
\hline A005 & -41.818 & 43.392 & 5.02501 & $\ldots$ \\
\hline A006 & -35.3672 & 37.7287 & 4.95222 & $\ldots$ \\
\hline A007 & -34.4579 & 35.6521 & 4.99769 & . \\
\hline A008 & -36.8554 & 39.0715 & 5.37675 & $\cdots$ \\
\hline A009 & -40.9368 & 42.0707 & 5.83162 & $\ldots$ \\
\hline A010 & -27.9413 & 28.3052 & 4.34862 & $\ldots$ \\
\hline A011 & -38.93 & 39.7303 & 5.69076 & $\ldots$ \\
\hline A012 & -29.3361 & 29.6703 & 4.58029 & . \\
\hline A013 & -34.9547 & 36.8532 & 4.99185 & $\ldots$ \\
\hline A014 & -24.3012 & 24.4997 & 3.74402 & $\ldots$ \\
\hline A015 & -32.4916 & 33.5219 & 5.04302 & $\ldots$ \\
\hline A016 & -35.6782 & 38.7783 & 5.02697 & $\ldots$ \\
\hline A017 & -36.438 & 39.4079 & 4.89321 & $\ldots$ \\
\hline A018 & -30.7231 & 31.0887 & 4.67771 & $\ldots$ \\
\hline A019 & -35.9598 & 38.3006 & 4.82963 & $\ldots$ \\
\hline A020 & -29.4345 & 29.7997 & 4.59663 & \\
\hline A021 & -39.3223 & 40.0984 & 5.7423 & \\
\hline$\ldots$ & $\ldots$ & $\ldots$ & $\ldots$ & $\ldots$ \\
\hline
\end{tabular}

Following the definition and algorithm proposed in Sect. 2 and Sect. 3, the chip's test data set is converted to a quality classifier matrix $\mathrm{Q}$ first. Then, a quality-spectrum is formed as Fig. 4 shows.

Figure 4 shows the quality-spectrum of a batch of chip. It includes 1000 chips which have 543 test parameters. The green pixels of the quality-spectrum display the distribution of the chip's perfect test data. And the red ones means the worst. The color bar on the right side of Fig. 4 displays the color transition from the green to red, which represent the chip's test data decreases from the best to the worst. That is, the defect chips and problematic parameters distribution could be observed intuitively. Furthermore, the quality-spectrum could be quantitative analyzed by calculating the its fault score of its parameter and chip as Eq. (13) and Eq. (14) shows. Figure 6 shows the fault score of each test parameter which displays 543 test parameter's fault comprehensively.

Through Fig. 5, it is easy to find the impact of overall test parameter to the chip's quality which is useful for the fault root tracing and fault diagnose. The chip's fault scores realize the comprehensive and quantitative judging the impact of overall test parameter. Obviously, the fault score of each chip represents its quality considering overall test parameters in this case. The overall chip's fault score is shown in Fig. 6.

As Fig. 6 shows, a lot of chip's fault score is high which indicates vital faults hidden that decrease the chip's quality. Through double check the chip's production process, the faults DO exist. These faults are stubble and hides deeply. It is difficult to be noticed without Fig. 6. 


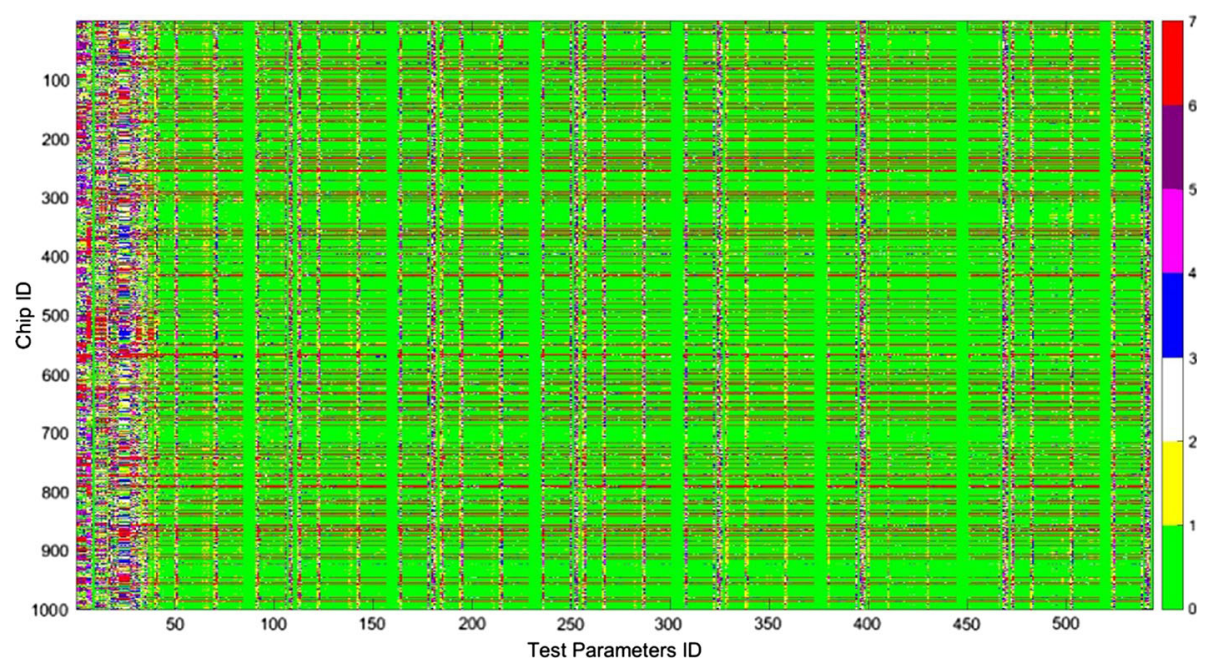

Fig. 4 The semiconductor chip's quality-spectrum

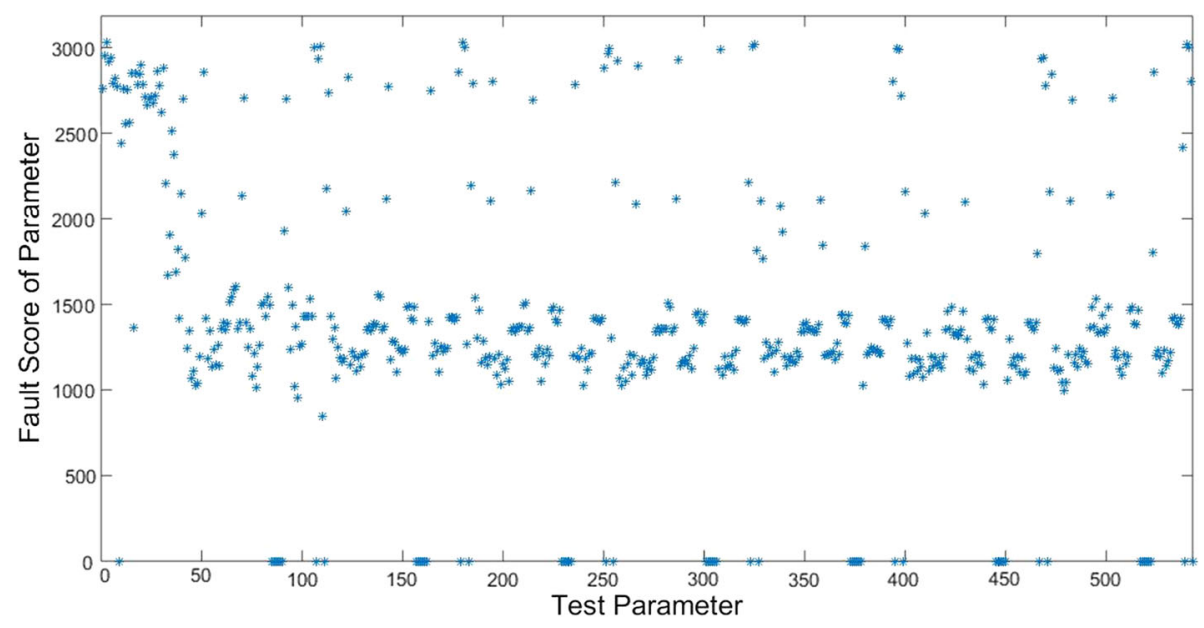

Fig. 5 Fault score of test parameter

\section{Conclusions}

The advantage of the quality-spectrum is its ability of dealing with multivariable series data set regardless the scale of the dimension. The chip's quality-spectrum displays the quality information of a high dimensional huge data by a digital color picture. Each test datum could be transformed to a special color. Through observing the quality-spectrum, it is easier to obtain useful information than a huge float data set because human's eyes are more sensitive to the change of the color than the float number. Therefore, NO parameter needs to be neglected which is a necessary step for any conventional method. Furthermore, analyzing quality-spectrum could use abundant methods of digital image processing. The proposed method bridge the field of chip's quality analysis with digital image processing. The use case 


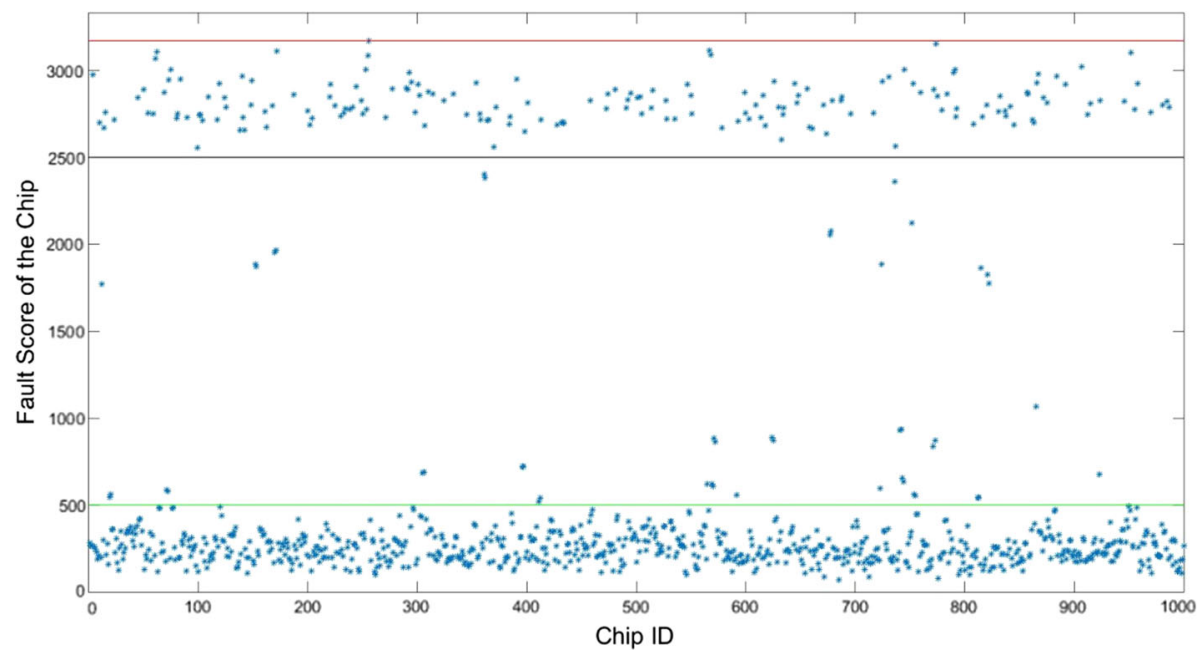

Fig. 6 Chip's fault score

of this paper using fault score to reveal the quality regular hidden inside a huge float data set is a preliminary exploration.

Acknowledgements This work is financial supported by the National Science and Technology Major Project of China (Grant No. 2016ZX03001002).

Open Access This article is distributed under the terms of the Creative Commons Attribution 4.0 International License (http://creativecommons.org/licenses/by/4.0/), which permits unrestricted use, distribution, and reproduction in any medium, provided you give appropriate credit to the original author(s) and the source, provide a link to the Creative Commons license, and indicate if changes were made.

\section{References}

Alcala, C. F., \& Qin, S. J. (2010). Reconstruction-based contribution for process monitoring with kernel principal component analysis. Industrial and Engineering Chemistry Research, 49, 7849-7857.

Amin, M. T., Imtiaz, S., \& Khan, F. (2018). Process system fault detection and diagnosis using a hybrid technique. Chemical Engineering Science, 189, 191-211.

Arroyo-Hernandez, J. (2016). Dimensionality Reduction Methods: Comparative Analysis of methods PCA, PPCA and KPCA. Uniciencia, 30(1), 115-122.

Bakdi, A., \& Kouadri, A. (2018). An improved plant-wide fault detection scheme based on PCA and adaptive threshold for reliable process monitoring: Application on the new revised model of tennessee eastman process. Journal of Chemometrics, 32(5), 2978.

Brodlie, K. W., Carpenter, L. A., Earnshaw, R. A., Gallop, J. R., Hubbard, R. J., Mumford, A. M., et al. (1992). Scientific visualization, techniques and applications. Berlin: Springer.

Burgas, L., Melendez, J., Colomer, J., Massana, J., \& Pous, C. (2018). N-dimensional extension of unfold-PCA for granular systems monitoring. Engineering Applications of Artificial Intelligence, 71, 113-124.

Cai, T. T., \& Zhang, L. J. (2016). Discussion of influential feature pca for high dimensional clustering. Annals of Statistics, 44(6), 2372-2381.

Chen, J. H., \& Liao, C. M. (2002). Dynamic process fault monitoring based on neural network and PCA. Journal of Process Control, 12, 277-289.

Chu, D. L., Wu, C. Q. (2016). On a pipeline-based architecture for parallel visualization of large-scale scientific data. In Proceedings of 45th International Conference on Parallel Processing Workshops (ICPPW 2016) pp. 88-97. 
Djauhari, M. A., Salleh, R. M., Zolkeply, Z., et al. (2017). On the reliability of shewhart-type control charts for multivariate process variability. AIP Conference Proceedings, 1842(1), 1-7.

Dong, D., \& McAvoy, T. J. (1996). Nonlinear principal component analysis-based on principal curves and neural networks. Computers \& Chemical Engineering, 20, 65-78.

Dong, Y. N., \& Qin, S. J. (2018). A novel dynamic PCA algorithm for dynamic data modeling and process monitoring. Journal of Process Control, 67, 1-11.

Dong, J., Zhang, K., \& Huang, Y. (2015). Adaptive total PLS based quality-relevant process monitoring with application to the tennessee eastman process. Neurocomputing, 154, 77-85.

Du, J. Z., Lu, W. G., Wu, X. H., Dong, J. Y., \& Zuo, W. M. (2018). L-SVM: A radius-margin-based SVM algorithm with LogDet regularization. Expert Systems with Applications, 102, 113-125.

Dyer, S. M., Laver, K., Friel, M., Whitehead, C., \& Crotty, M. (2017). The diagnostic accuracy of the kimberley indigenous cognitive assessment (KICA) tool: A systematic review. Australasian Psychiatry, 25(3), 282-287.

Foo, P. Y., Lee, V. H., Tan, G. W. H., \& Ooi, K. B. (2018). A gateway to realising sustainability performance via green supply chain management practices: A PLS-ANN approach. Expert Systems with Applications, 107, 1-14.

Galmeanu, H., Sasu, L. M., \& Andonie, R. (2016). Incremental and decremental SVM for regression. International Journal of Computers Communications \& Control, 11(6), 755-775.

Gao, X., Gao, J., Sun, K., et al. (2016). Online fault diagnosis of modern process industry system based on color-spectrum. Journal of Shanghai Jiaotong University (Science), 23(5), 621-628.

Ge, Z. Q., Yang, C. J., \& Song, Z. H. (2009). Improved kernel PCA-based moni-toring approach for nonlinear processes. Chemical Engineering Science, 64, 2245-2255.

Gharahbagheri, H., Imtiaz, S. A., \& Khan, F. (2017). Root cause diagnosis of process fault using KPCA and bayesian network. Industrial and Engineering Chemistry Research, 56(8), 2054-2070.

Habchi, B., Kassouf, A., Padellec, Y., et al. (2018). An untargeted evaluation of food contact materials by flow injection analysis-mass spectrometry (FIA-MS) combined with independent components analysis (ICA). Analytica Chimica Acta, 1022, 81-88.

He, H., \& Tan, Y. H. (2018). Pattern clustering of hysteresis time series with multivalued mapping using tensor decomposition. IEEE Transactions on Systems Man Cybernetics-Systems, 48(6), 993-1004.

Iglesias, C., Sancho, J., Pineiro, J. I., et al. (2016). Shewhart-type control charts and functional data analysis for water quality analysis based on a global indicator. Desalination and Water Treatment, 57(6), 2669-2684.

Jemwa, G. T., \& Aldrich, C. (2005). Improving process operations using support vector machines and decision trees. AIChE Journal, 51, 526-543.

Jiang, J., Jiang, X., Li, J., Liu, Y., \& Yan, W. (2017). Spatial quantile estimation of multivariate threshold time series models. Physica A-Statistical Mechanics and its Applications, 486, 772-781.

Kai, Sun, Jianmin, Gao, Zhiyong, Gao, Hongquan, Jiang, \& Gao, Xu. (2015). Plant-wide quantitative assessment of a process industry system's operating state based on color-spectrum. Mechanical Systems and Signal Processing, 60-61, 644-655.

Lee, J. M., Qin, S. J., \& Lee, I. B. (2006). Fault detection and diagnosis of multi-variate process based on modified independent component analysis. AIChE Journal, 52, 3501-3514.

Lee, J. M., Qin, S. J., \& Lee, I. B. (2007). Fault detection of non-linear processes using kernel independent component analysis. Canadian Journal of Chemical Engineering, 85, 526-536.

Lee, J. M., Yoo, C. K., Choi, S. W., Vanrolleghem, P. A., \& Lee, I. B. (2004a). Non-linear process monitoring using kernel principal component analysis. Chemical Engineering Science, 59, 223-234.

Lee, J. M., Yoo, C. K., \& Lee, I. B. (2004b). Statistical process monitoring with independent component analysis. Journal of Process Control, 14, 467-485.

Li, H. Q., Liang, H., Miao, C. J., et al. (2016). Novel ECG signal classification based on KICA nonlinear feature extraction. Circuits Systems and Signal Processing, 35(4), 1187-1197.

Ma, L. R., Song, D. D., Liao, L. J., \& Wang, J. G. (2017). PSVM: a preference-enhanced SVM model using preference data for classification. Science China-Information Sciences, 60(12), 122103.

Mania Navi; Nader Meskin; Mohammadreza Davoodi. (2018). Sensor fault detection and isolation of an industrial gas turbine using partial adaptive KPCA. Journal of Process Control, 64, 37-48.

McCormick, B. H., Defanti, T. A., \& Brown, M. D. (1987). Visualization in scientific computing. Computer Graphics, 21(6), 1-14.

Montgomery, D. C. (2005). Introduction to statistical quality control (5th ed.). New York: Wiley.

Nguyen, H. N., \& Lee, H. H. (2018). An effective SVM method for matrix converters with a superior output performance. IEEE Transactions on Industrial Electronics, 65(9), 6948-6958.

Nomikos, Paul, \& MacGregor, John F. (1995). Multivariate SPC charts for monitoring batch processes. Technometrics, 37(1), 41-59. 
Tong, W. D., Xie, W., Hong, H. X., Fang, H., Shi, L. M., Perkins, R., et al. (2004). Using decision forest to classify prostate cancer samples on the basis of SELDI-TOF MS data: Assessing chance correlation and prediction con-fidence. Environmental Health Perspectives, 112, 1622-1627.

Tootooni, M. S., Rao, P. K., Chou, C. A., \& Kong, Z. Y. (2018). A spectral graph theoretic approach for monitoring multivariate time series data from complex dynamical processes. IEEE Transactions on Automation Science and Engineering, 15(1), 127-144.

Wang, X. G., Huang, L. W., \& Zhang, Y. W. (2017). Modeling and monitoring of nonlinear multi-mode processes based on similarity measure-KPCA. Journal of Central South University, 24(3), 665-674.

Wang, J., Zhang, Y., Cao, H., \& Zhu, W. (2012). Dimension reduction method of independent component analysis for process monitoring based on minimum mean square error. Journal of Process Control, 22, $477-487$.

Wold, S., Esbensen, K., \& Geladi, P. (1987). Principal component analysis. Chemometrics and Intelligent Laboratory Systems, 2, 37-52.

Zeng, G. M., Li, X. D., Jiang, R., Li, J. B., \& Huang, G. H. (2006). Fault diagnosis of WWTP based on improved support vector machine. Environmental Engineering Science, 23, 1044-1054.

Zhang, Y. W. (2008). Fault detection and diagnosis of nonlinear processes using improved kernel independent component analysis(KICA) and support vector machine (SVM). Industrial and Engineering Chemistry Research, 47, 6961-6971.

Publisher's Note Springer Nature remains neutral with regard to jurisdictional claims in published maps and institutional affiliations. 